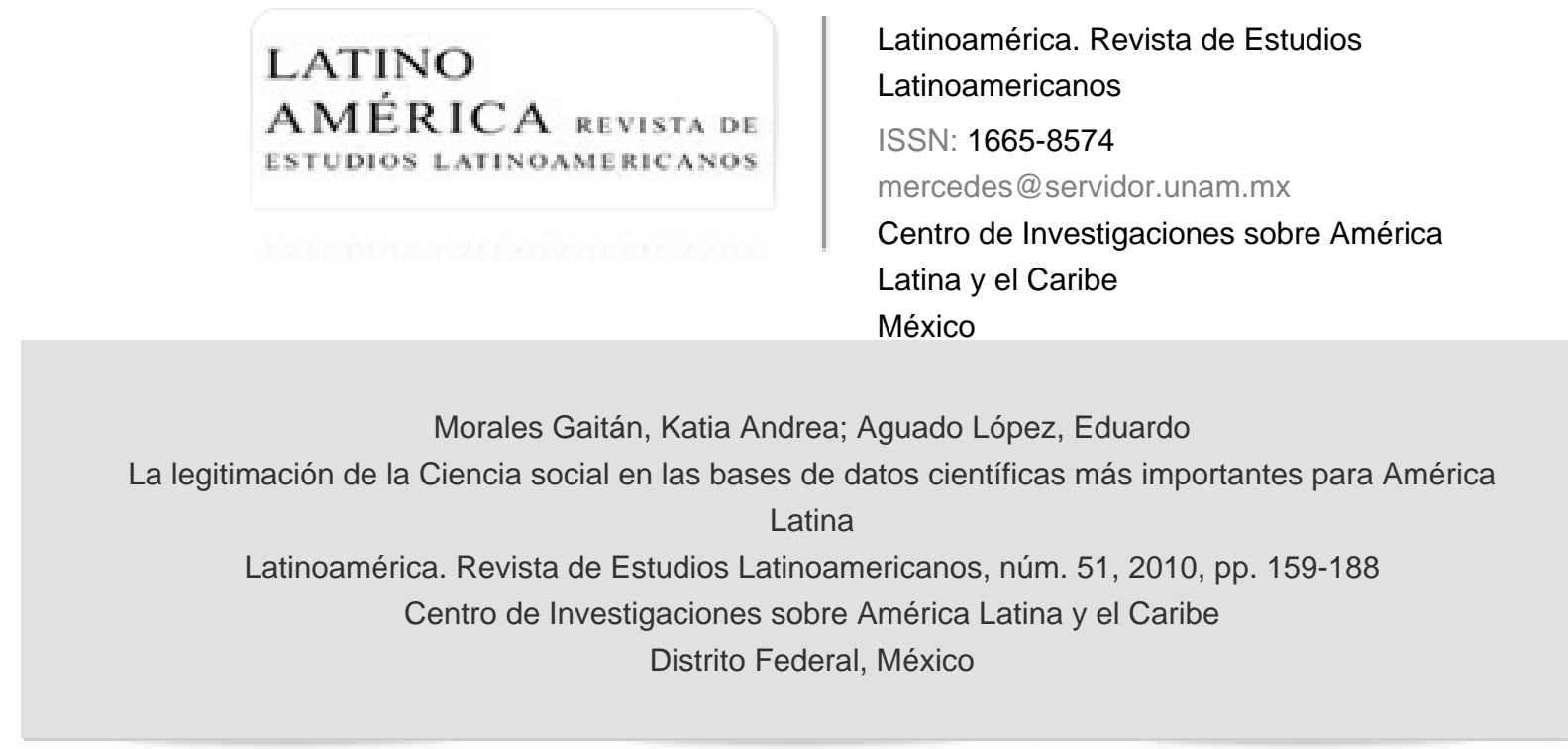

Disponible en: http://www.redalyc.org/articulo.oa?id=64015153008

Cómo citar el artículo

- Número completo

- Más información del artículo

Página de la revista en redalyc.org

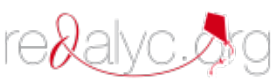

Sistema de Información Científica

Red de Revistas Científicas de América Latina, el Caribe, España y Portugal Proyecto académico sin fines de lucro, desarrollado bajo la iniciativa de acceso abierto 


\title{
La legitimación de la Ciencia social en las bases de datos cientí- ficas más importantes para América Latina
}

\author{
Katia Andrea Morales Gaitán* y Eduardo Aguado López***
}

Resumen: Se analizan desde la Sociología de la ciencia tres ejes de su campo. El primero, rescata la epistemología de la producción científica social. El segundo, trata la construcción de la legitimidad de los científicos a través de sus publicaciones. El tercero, refiere el estudio de los indicadores más importantes para América Latina generados por la Cienciometría. Se concluye que la incorporación de las Tecnologías de Información y Comunicación son canales a través de los cuales se legitiman los resultados de investigación, productividad y visibilidad entre las comunidades científicas.

PalaBRAS Clave: Sociología de la ciencia, Legitimidad, Ciencia social, Producción científica, Cienciometría.

ABSTRAC: We analyze, from the point of view of the Sociology of Science three constituent axes of its field. The first level rescues the epistemology of the social scientific production. The second focuses on the construction of the legitimacy on scientists through their publications. Finally, we refer throught the most important indicators for Latin-America, generated in the scientific databases. Concluding that the use of Technologies of Information and Communication are channels that legitimize the results of research and productivity in the scientific communities.

KEy Words: Sociology of Science, Legitimacy, Social Science, Scientific production, Scienciometrics.

Asociación Latinoamericana de Sociología, UAEM (katiakatiush@hotmail.com).

** Facultad de Ciencias Políticas y Sociales, uaEm (eaguado@uaemex.mx). 
INTRODUCCIÓN

L

a ciencia ha desempeñado un papel decisivo en el desarrollo económico y social de las naciones mundiales desde hace más de dos siglos, ${ }^{1} \sin$ embargo, es reciente el surgimiento de disciplinas enfocadas al estudio de la propia actividad científica. La Sociología de la ciencia ha investigado con profundidad los procesos de producción y comunicación científica, especialmente porque retoma la ciencia y la tecnología como fuentes de información contemporánea, útiles para la evaluación científica, aun cuando ha estudiado a la ciencia, ha enfatizado los aspectos epistemológicos y ha discutido sobre la objetividad y neutralidad de su naturaleza, en este sentido la verdad científica reposa sobre diversos parámetros, donde el enfoque ha sido guiado por la teoría crítica y práctica en la Sociología de la ciencia, área que cuestiona la construcción e institucionalización social de la ciencia.

Al mismo tiempo, la incorporación de nuevas tecnologías de la información y las comunicaciones (TIC's) sitúan al ser humano en contextos de cambios fundamentales en la manera de producir, pero principalmente de comunicar y publicar investigaciones científicas. La comunicación científica se ha convertido en un sistema complejo en el que aparecen factores, individuos y mecanismos que apuestan por una nueva organización y almacenamiento de la información, dispositivos que además se perfilan como evaluadores de producción científica. ${ }^{2}$ Estos cambios en la comunicación se traducen en modificaciones de las nuevas formas de legitimación y posicionamiento de los científicos.

\footnotetext{
${ }^{1}$ Las diferencias de inversión en ciencia y tecnología de acuerdo al piB de los países es uno de los aspectos fundamentales para el desarrollo de sus sociedades. Por ejemplo Suecia y Japón invierten 3.0\%, Estados Unidos 2.4\%, los países del sureste asiático llamados "tigres" han invertido hasta $5.0 \%$ de su PIB total, logrando desarrollar y mejorar la calidad de vida de sus habitantes en menos de 40 años, situación que contrasta en América Latina, donde el promedio invertido es de $0.7 \%$, para el caso específico de México sólo destina $0.01 \%$ de su PIB. Raimundo Javier Páez Abello et al., "iSon la ciencia y la tecnología un instrumento de desarrollo? Un análisis para el caso de América Latina", en Investigación y Desarrollo, vol. 9, núm. 1, Barranquilla, Colombia, Universidad del Norte, julio, p. 372.

2 Julie M. Hurd, "Scientific Communication: New Roles and New Players", en Emerging Issues in the Electronic Environment: Challenges for Librarians and Researchers in the Sciences, Londres, Co published as The Haworth Information Press, 2004, p. 5.
} 
El paradigma científico denominado "corriente de la ciencia principal" considera la publicación de artículos en revistas indexadas como el indicador más representativo de la capacidad de producción de un científico y, en consecuencia, de evaluación científica global, porque para lograr publicar en una revista es preciso llevar a cabo el proceso de arbitraje por pares ciegos, así como la revisión del documento por parte de los comités editoriales, órganos que verifican y garantizan la calidad de un trabajo. Desde hace varios años se considera que el proceso de arbitraje es garante de calidad e innovación. Práctica tradicional a la que las ciencias naturales han recurrido para comunicar, difundir y evaluar sus investigaciones; es a través de la publicación de artículos que las ciencias duras han abierto las posibilidades de discusión y superación de los resultados. A partir de los índices que la Cienciometría arroja, podemos identificar a los autores que tienen una mayor producción, saber con quiénes trabajan, sus grupos de trabajo y de esta forma conocer las colaboraciones, tanto institucionales como internacionales de todas las áreas científicas. ${ }^{3}$

De esta forma, al tener claro que las ciencias naturales han comunicado y evaluado sus resultados hasta convertirse en el eje principal de su lógica de legitimación, se pregunta si estas formas y métodos se podrían incorporar a las ciencias sociales y humanidades. ¿Qué ventajas tendría adoptarlos? Más allá de la crítica marginal, ¿qué estamos haciendo desde las ciencias sociales para hacer visibles nuestras investigaciones? ¿Por qué para el paradigma de la corriente principal, América Latina es un punto de interés para incorporar su producción científica? ¿Cómo responde la comunidad científica latinoamericana ante estas instancias? Éstas y otras preguntas son tratadas en este texto, algunas respuestas se han logrado inferir, otras se abren para futuros debates y alternativas.

${ }^{3}$ La problemática de otras formas de comunicación científica es la poca sistematización de su impacto. Por ejemplo, la publicación de libros no evalúa sus contenidos y se considera - en muchos casos - una acción aislada cuya circulación se limita a la institución que lo haya publicado, sin trascender a mayores ámbitos internacionales. Por su parte, no todos los congresos compilan los trabajos presentados, lo que provoca que el seguimiento de las investigaciones y sus autores sea limitado y con claros sesgos. 
En otra perspectiva, en autores como Pierre Bourdieu, Olivier Martín, Robert Merton y Jeffrey Alexander provenientes de distintas escuelas sociológicas, francesa e inglesa, existen puntos de coincidencia acerca de la legitimidad de los científicos. Señalan, grosso modo, que el consenso de sus comunidades, el sistema de relaciones y su participación en redes y grupos afines son vitales para su posicionamiento y legitimidad. Si se quiere ver desde el sentido de la Cienciometría son los indicadores, la publicación y coautoría de artículos en revistas indexadas, y el sometimiento de la evaluación por pares de los trabajos los que se perfilan como procesos legitimadores y de calidad en las investigaciones.

Por su parte, la propuesta de la corriente de la ciencia principal, que representa las bases de datos o indexadores, apuesta porque los aportes deben restringirse a las mismas reglas, sea el caso de ciencias sociales o naturales: que la comunicación de sus resultados sea a través de canales legitimados; que haya aceptación de los trabajos por parte de la comunidad correspondiente, aunque reconoce que dicha aceptación no se encuentra exenta de tensiones, conflictos e intereses.

En la actualidad, hay dos empresas que se han apropiado de los canales de legitimación de comunicación de resultados (Scopus en Europa, Thompson Reuters, en Estados Unidos) al agrupar más de 10000 revistas de todas las áreas, aducen que en ellas es donde se presentan los aportes y que en el resto de las otras revistas, los resultados no contribuyen de forma central, por lo tanto compiten entre sí para obtener el estatus de principales portavoces de la comunicación y difusión científica, legitimando los procesos que se emiten por este canal.

De acuerdo al Journal Citation Index del Thompson Reuters, los espacios más relevantes de investigación sociológica se concentran en bases de datos anglófonas como la Sociological Abstracts y a través de revistas como el American Journal of Sociology de la Universidad de Chicago, la American Sociological Review de la Asociación Americana de Sociología y el British Journal of Sociology publicado por la London School of Economics. Gran parte de la literatura científica proveniente de Latinoamérica y España está fuera de estas bases de datos, por lo que han surgido proyectos como scielo y Redalyc que buscan reunir las revistas de mayor calidad de esta región, con una perspectiva distinta a la del 
mercado, es decir, la del acceso abierto. scielo contiene prioritariamente las revistas de ciencias naturales y exactas, mientras que Redalyc incluye de preferente manera revistas de ciencias sociales y humanidades, con la finalidad de evaluar la producción científica regional en la esfera global.

Planteamos que no sólo los factores cuantitativos provenientes de las bases de datos o indexadores de revistas son los que otorgan legitimidad a los científicos, sino que también las redes sociales a las que se les vincule; su poder político y social desempeñan un papel importante en dicho proceso. Para articular la problemática, se desarrollan nuestros argumentos en tres tiempos. Primero, se retomarán las pautas epistemológicas para este trabajo. Se referirá a los aportes de los precursores de la sociología de la ciencia como Robert Merton. Haremos especial referencia a la noción de campo de Pierre Bourdieu y su perspectiva sobre la producción científica. Este apartado se nutrirá también de las contribuciones que ofrece a la filosofía de la ciencia Jeffrey Alexander, autor que, asimismo, permitirá dar cuenta de los distintos campos de legitimación científica.

Posteriormente, aclararemos el concepto de legitimación de la ciencia y sus manifestaciones abstractas y concretas. Después tendremos un espacio para la discusión de la problemática de la legitimación de la Ciencia social y por último, se expondrá el surgimiento y la evolución de la Cienciometría como línea de investigación estudiada por la Sociología de la ciencia. Aunado a este tema, se analizarán cuatro bases de datos: Thompson Reuters, Scopus, Redalyc y scielo. Estos proyectos permitirán ejemplificar cómo los distintos campos científicos construyen sus propios dispositivos de legitimación.

Para concluir, se propone una reflexión global a fin de simplificar cómo se constituye la legitimidad de la producción científica en campos de tradición particular como el de las ciencias sociales en contextos como América Latina.

\section{BASES EPISTEMOLÓGICAS PARA UNA SOCIOLOGÍA DE LA CIENCIA}

En este apartado es fundamental referirse a los estudios de Robert Merton, quien desde los años treinta y cuarenta del siglo pasado se esforzó por definir una Sociología específica para la ciencia. 
Las normas que Merton logró identificar como patrones de comportamiento de los científicos son: el universalismo, el comunismo, el desinterés y el escepticismo, asimismo remarcó hechos que existen en la ciencia, como el fraude, las disputas por descubrimientos, el plagio, entre otras. La crítica a Merton radica en que sus cuatro normas no son una práctica constante entre la totalidad de las comunidades científicas en un ámbito mundial, y que es evidente la existencia de intereses políticos y económicos en los descubrimientos de la ciencia; que no todos los investigadores son escépticos y que, en realidad, el mundo contemporáneo privilegia al individuo por encima de la comunidad.

Por otro lado, para Bourdieu el capital científico es una especie particular de capital simbólico, fundado en el conocimiento y reconocimiento, que otorgan los pares competidores del campo científico específico. A partir de ello, el campo científico - como cualquier otro- es un espacio que se constituye a partir de relaciones de fuerza, de luchas, de intereses concretos de los científicos, y de una serie de tensiones entre los recién llegados y los que ya conforman un grupo científico. Por su parte, el concepto de capital cultural (heredado o adquirido) del autor resulta importante para reconocer tanto las calidades, como las cualidades de un científico. En el discurso de Bourdieu, la ciencia es un campo condicionado por las producciones sociales: "se trata de un estado determinado por las leyes del mercado". ${ }^{4}$ La reproducción y legitimación de un científico es todo un proceso que implica su aceptación por parte de los colegas, la cooperación y, finalmente, la evaluación de otros científicos.

Por lo tanto, en cada disciplina se van entretejiendo redes de colaboración, las cuales conforman distintos investigadores, de uno o varios países, con el fin de compartir su labor para hacerse más productivos, las redes intercambian y desarrollan en conjunto información de manera estructurada, lo que da como resultado una jerarquización de acuerdo al rol y a la participación de cada científico. Las redes pueden ser cerradas o bien abiertas cuando el trabajo se comparte con varias disciplinas o instituciones.

${ }^{4}$ Pierre Bourdieu, Algunas propiedades de los campos en sociología y cultura, México, Conaculta, 1990, p. 135. 
Una condición vital del concepto de campo es que exista un juego y jugadores que estén dispuestos a respetar las reglas que moderan el juego. En el campo de la ciencia, el juego es la producción científica y la creación de nuevos conocimientos, los jugadores son los científicos y las reglas del juego se relacionan con la pertinencia de una investigación, las metodologías empleadas para llevarla a cabo, con las formas establecidas para su difusión, comunicación y discusión. Tal es el caso de los canales de comunicación y revisión por pares. A estos patrones, Bourdieu les ha llamado habitus y señala un claro ejemplo: "Un habitus de filólogo es a la vez un oficio, un conjunto de técnicas, de referencias [...] propiedades que dependen a su vez de la historia de la disciplina, de su jerarquía en las disciplinas, y que son a la vez condiciones para que funcione el campo".

En la historia de la ciencia, la revisión por pares y la publicación de artículos en revistas se han legitimado con base en sus propios mecanismos de producción. ${ }^{6}$

Al mismo tiempo, los campos poseen la característica de ser estructurados. Dicha estructura responde al estado en que la lucha derivará al poseer el monopolio de la legitimación. Así la lucha adquiere un nuevo sentido, el apropiarse de los canales validados. Siguiendo la perspectiva de Bourdieu, el estado más "puro" de la ciencia es en realidad un dominio social como cualquier otro, con sus relaciones de fuerza, sus monopolios, entre otros.

El funcionamiento mismo de un dominio científico supone una forma y existencia específica de interés, sean éstos económicos, políticos e incluso sociales. Las prácticas científicas no aparecen como desinteresadas, sino por el contrario, éstas son realizadas en el marco de beneficios particulares de cada campo. En ciertos casos, los avances e innovaciones científicos se convierten en productos que más tarde se comercializarán y de esta forma se obtienen ganancias en el mundo económico. Un claro ejemplo lo tenemos en la industria arma-

${ }^{5}$ Loc. cit.

${ }^{6}$ Sin embargo aún quedan varios aspectos por cambiar, tales como los procesos perversos de la manipulación social y de intereses en la ciencia, así como la participación efectiva de los comités editoriales. 
mentista que desarrolla cada día más tecnología y comunicaciones, con la finalidad de dar más poder y control a quienes sean capaces de comprar éstas. ${ }^{7}$

En este sentido, cada campo científico sigue las regularidades que le permitan funcionar con base en su propia historia y legalidad específica, en particular a través de sus mecanismos de comunicación y recompensas. Bourdieu, en su obra Homo Academicus, estudia los mecanismos de promoción de los hombres que viven en y desde la academia. Hace un estudio concreto sobre L'École des Hautes Études de París y señala: "el prestigio académico" no se relaciona específicamente con el estímulo científico o intelectual. En todo caso, significa que el aplauso y reconocimiento académicos suelen darse a quienes saben cómo manejarse en el sistema de mentores y utilizan con estrategia sus privilegios heredados del capital cultural que poseen. Por tanto, el renombre científico implica creación original, capacidad aguda de crítica, reflexión y otras capacidades. Mientras que el poder institucional y el privilegio académico resultan de la prueba más genuina de obsequium, "respeto incondicional por los principios fundamentales del orden establecido". ${ }^{8}$ Por supuesto el medio académico es mucho más complejo, pero este análisis nutre la perspectiva de la legitimación en específico de un caso en ciencias sociales.

Al seguir las reflexiones de Jeffrey Alexander, el desarrollo y la crisis de los programas de investigación social deben ser entendidos como líneas de tradición explicativa que funcionan como unidades críticas de análisis, las cuales buscan la acumulación del conocimiento sobre una determinada área de lo social. Sin embargo, el conocimiento social avanza y decae de forma distinta a lo que ocurre en

${ }^{7}$ Una perspectiva compartida la encontramos en Michaell Pollak, en su artículo "Proyecto científico, carrera profesional y estrategia política", en el que analiza la trayectoria y la biografía de Paul F. Lazarsfeld. Su análisis radica en que la producción científica y las opiniones políticas se encuentran mediatizadas por las formas de organización de la investigación. Por tanto, la selección de los objetos sociológicos, métodos de investigación estadísticos y matemáticos de Lazarsfeld fueron racionalizados por una dinámica de pago o económica; en este caso, de la Compañía Ford, quien le financió varios proyectos que le permitieron posicionarse en el mercado de la investigación americana y, posteriormente, europea. Michael Pollak, "Projet scientifique, carrière professionnelle et stratégie politique", en Actes de la Recherche en Sciences Sociales, vol. 55, núm. 55, París, 1994, pp. 54-63.

${ }_{8}^{8}$ Pierre Bourdieu, Homo Academicus, trad. de Peter Collier, Cambridge, Polity Press, 1988, p. 87. 
las ciencias duras. Los programas de investigación, de esta forma, deben ser comprendidos como un ámbito continuo de reflexiones generales, abstractas y metafísicas, por un lado; pero por el otro, tienen un rostro concreto, empírico y factual.

Las ciencias sociales - y en particular la sociología - pueden ser entendidas como conjuntos de tradiciones a las cuales regularmente se les denomina escuelas. De esta forma, se les puede definir como un discurso racional-pragmático en distintos multiniveles acerca de la sociedad y sus unidades constitutivas, con patrones y direcciones que son determinados por las distintas tradiciones de las disciplinas de las ciencias sociales. Los elementos de esta definición, como el propio Alexander argumenta, forman una paradoja y no una contradicción, en la medida en que las tradiciones son patrones de percepción y comportamiento que se siguen, en una primera instancia, no por su racionalidad intrínseca, sino porque son inherentes a su pasado.

Las escuelas de investigación y su historia se definen a sí mismas por sus núcleos teóricos que son muy resistentes al cambio, pero las áreas que las rodean -en algunos casos- son sustanciales, y se encuentran en constante variación. En términos típico-ideales, los cambios en las zonas periféricas de los núcleos teóricos de las costumbres de investigación pueden ser concebidos como procedentes de tres líneas: elaboración, proliferación y revisión. La estrategia principal que han seguido las distintas escuelas para poder difundir y legitimar sus trabajos es la creación de sus propios canales de comunicación, en especial a través de la fundación de sus propias revistas.

La reconstrucción teórica, por su parte, lleva a que las diferencias con la tradición sean explícitas y se abre a otras, también, de forma explícita. La reconstrucción puede revivir una tradición teórica e incluso crear la oportunidad para que nazcan nuevas. Pero también las tradiciones pueden ser deconstruidas. Esto no sucede porque el núcleo o las zonas periféricas hayan sido falsificadas, ocurre porque sus compromisos han sido deslegitimados a los ojos de la comunidad científica; en este sentido, deslegitimación implica retirar la confianza a los compromisos del núcleo.

Elaboración, proliferación y revisión no describen el desarrollo y grado real del avance científico en ciencias sociales. Un nuevo planteamiento teórico, por 
ejemplo, habla de un cambio en la sensibilidad científica, al poner diferentes cuestiones en la mesa de discusión y agregar un apremio fundamental en la creación de diferentes discursos. De este modo es que Jeffrey Alexander sugiere que se debe hablar de "movimientos sociales científicos y desarrollo científico social".

Así, el principal motor del cambio científico es pragmático: conflicto y competencia entre tradiciones. Por consecuencia, la noción de revoluciones científicas aportada por T. Kuhn, resulta vital para superar los paradigmas existentes en la ciencia normal. Con frecuencia, la competencia entre escuelas es subestimada, pero el rol que desempeñan es muy estimulante en el marco de la actividad hacia los programas de investigación. La competencia ocurre en dos géneros discursivos y sus herencias.

El primer tipo de competencia tiene que ver con las disputas al interior de una tradición con sus categorías residuales; con la amplitud analítica y con la empírica, así como con la forma en cómo se leen e interpretan a los teóricos clásicos. El segundo tipo de competencia se da en el nivel de los programas de investigación, una competencia que se encuentra organizada alrededor del intento de los rivales por explicar las estructuras empíricas y los procesos vinculados al análisis de la tradición o la escuela de investigación.

Hay que destacar que, como apunta Alexander, una tradición avanza cuando los temas de sus declaraciones son juzgadas como relativamente superiores, o comparables con el trabajo de investigación producido por otras escuelas. La mejor defensa de una tradición bajo ataque de otra es la de articular y defender las bases analíticas de su programa de investigación. Como es de esperarse, "la competencia genera ganadores y perdedores". ${ }^{9}$ Este proceso es sociológico más que epistemológico, porque la competencia implica una movilización importante, no sólo de recursos intelectuales y argumentativos, sino también materiales y económicos que permiten que un programa de investigación se consolide.

Ahora bien, desde una visión contemporánea de la Cienciometría americana, el hombre actual se encuentra ante una epistemología que se ha reproducido en formas tecnocráticas de las instituciones privadas y públicas; traducida

9 Jeffrey Alexander, Real Civil Societies: Dilemmas of Institutionalization, SAGE, 1998, p. 42 (traducción nuestra). 
en modelos de productividad, crecimiento y desarrollo económico. Esta perspectiva establece que la información sistematizada permite la evaluación, es decir, la identificación jerárquica de la contribución medida en producción (artículos) o impacto (citas). Desde sus comienzos, la Cienciometría se caracteriza por ser un estudio que permite analizar, observar y mesurar la actividad científica. Este término surgió hace más de dos décadas y se vincula directamente a la revista Scientometrics. A partir de la Cienciometría, la sociología de la ciencia ha exportado sus índices numéricos para generar esquemas y estudiar el funcionamiento de las instituciones científicas.

A través de un sistema meritorio, los científicos se ven recompensados y legitimados por su trabajo, al obtener medallas, premios especiales, puestos directivos, adscripción a sociedades honoríficas, premios Nobel, entre otros. Lo que se percibe es que la legitimación científica cuenta con un discurso organizado, que al contrastar en la práctica, encontramos algunos sesgos, como lo menciona Bourdieu: al haber establecido que hay una correlación entre la cantidad de publicaciones y los índices de reconocimiento, los autores se preguntaron si la mejor medida de excelencia científica es la cantidad o la calidad de las producciones. De esta forma estudiaron la relación entre los outputs cuantitativos y cualitativos de 120 físicos: hay una correlación, sin embargo algunos físicos publican muchos artículos de poca importancia. ${ }^{10}$

Nótese que es en las ciencias naturales y exactas donde nace la mayor parte de ellas. Mientras que las ciencias sociales, extraordinariamente, aportan al mundo en este sentido. Otro reto que desafía a la Cienciometría está en el tema de los países que encabezan las listas de mayor productividad e innovación, ya que de nueva cuenta observamos desigualdades profundas entre las diferentes regiones del mundo.

10 “[...] Ayant établi qu' il y a une corrélation entre la quantité de publications et les indices de reconnaissance, les auteurs se demandent si la meilleure mesure de l' excellence scientifique est la quantité ou la qualité des productions. Ils étudient donc la relation entre les outputs quantitatifs et qualitatifs de 120 physiciens: il y a une correlation mais quelques physiciens publient beaucoup d' articles de peut importance..." (Cole \& Cole, citado en Pierre Bourdieu, Les usages sociaux de la science, París, inRA Editions, 1997, p. 82). 
A partir de los datos obtenidos de 5500 encuestas que se aplicaron en Japón y Estados Unidos, Walsh y Nagaoka. señalan en su artículo "¿Quién inventó?", que son los científicos japoneses y americanos quienes lideran la productividad de patentes. Afirman que tienen bastantes similitudes respecto al tipo de organizaciones en las que trabajan; los inventores provienen en mayor grado de las universidades, así como la constante distribución en inventos a lo largo de diferentes clases de tecnologías. ${ }^{11}$

Otra interesante experiencia que se vincula a este problema la encontramos en el artículo de Wayt Gibbs, "Ciencia del Tercer Mundo", en el que afirma que el nombre de los países donde se crea y produce ciencia sigue siendo un factor determinante para que los legitimadores de estas producciones tengan su propio reconocimiento.

Lo que da como resultado una visión donde los países del tercer mundo y sus científicos no tenemos aportes significativos a los ojos de los países industrializados [...]. Algunos científicos que se transfirieron de un país industrializado a otro en vía de desarrollo constataron que la modificación de su dirección postal modificaba la apreciación de sus revisores. ${ }^{12}$

\section{LEGITIMIDAD EN LAS CIENCIAS SOCIALES}

El análisis de poder y legitimidad de los intelectuales es un tema difícil y ambiguo, debido a dos razones; primero por la naturaleza de la comunidad, y segundo, por tratarse de sujetos que existen en nuestra propia posición dentro de un grupo de investigadores. Hemos partido de la premisa de que la crítica propositiva construye mejores horizontes a la actividad científica. La variedad de enfoques y metodologías aplicadas a los sujetos y objetos de estudio en las ciencias sociales han generado controversias respecto a su estatus y relevancia científica. Corrientes y escuelas relacionadas con nombres como el positivismo, la hermenéutica, la fenomenología, el interaccionismo simbólico, el funcionalismo,

\footnotetext{
${ }^{11}$ John Walsh \& Sadao Nagaoka, "Who Invented?: Evidence from the Japan-US Inventor Survey", en RLETI Discussion Paper Series, vol. 9-E, núm. 34, pp. 1-70.

${ }^{12}$ Wayt Gibbs, "Ciencia del Tercer Mundo”, en Investigación y Ciencia, núm. 231, pp. 70-79.
} 
el estructuralismo, el post-estructuralismo han generado diversos esquemas de análisis, tanto a los sujetos como a los objetos estudiados.

Esta realidad histórica, dentro del proceso de consolidación de las ciencias sociales, le ha otorgado cierta debilidad respecto a su legitimidad y formalidad empírico-científica.

Entre otros problemas, los teóricos de la sociedad, abordan los siguientes temas: el status de las ciencias sociales, especialmente en relación a la lógica de las ciencias naturales; la naturaleza de las leyes y generalizaciones que pueden establecerse; la interpretación de la agencia humana y el modo de distinguirla de los objetos y acontecimientos naturales; y el carácter o forma de las instituciones humanas. ${ }^{13}$

Ante tan distintas temáticas de estudio se observa que paradójicamente en las ciencias sociales, hoy en día, el consenso con nuestros colegas resulta poco probable, - -aunque estudiemos un mismo tema de investigación, las divergencias y enfrentamientos por el planteamiento de la problemática, las metodologías y los resultados obtenidos-y es una realidad. La afirmación de Kuhn, quien estudió por un año una comunidad de científicos sociales es una evidencia: "me asombré ante el número y el alcance de los desacuerdos patentes entre los científicos sociales, sobre la naturaleza de problemas y métodos cientíicos aceptados". ${ }^{14}$

A través de esta afirmación, observamos que la Ciencia social aún no ha encontrado consenso universal sobre sus objetos de estudio, métodos de investigación o el tipo ideal de Ciencia social, situación que desemboca en una serie de desequilibrios de los procesos y tradiciones de legitimación respecto a las ciencias naturales. Frente a este contexto, nos preguntamos icómo reunir a las cien-

\footnotetext{
${ }^{13}$ En los últimos 30 años, la Ciencia social ha sido impregnada por la filosofía de la Ciencia natural. De esta forma el empirismo lógico que representaron científicos como Kuhn, Lakatos, Hesse, entre otros, ha sido rebasado por una perspectiva que niega la existencia de observaciones neutrales. Rebate la canonización de la generación de leyes generales derivadas del pensamiento deductivo y, a su vez, se consolida la perspectiva de una ciencia más interpretativa, la cual choca con la propuesta. O en términos del propio Giddens, se "desencanta" de las teorías de la corriente de la ciencia principal. Anthony Giddens et al., trad. de Jesús Alborés, La teoría social boy, Madrid, Alianza Universidad, 1990, p. 9.

${ }^{14}$ T. S. Kuhn, La estructura de las revoluciones cientificas, México, FCE, 1983, p. 13.
} 
cias sociales en un parámetro de desempeño además de la citación? ¿Cómo podemos tener en claro quiénes son los científicos más legítimos? Cuando hablamos de legitimación de la ciencia, queda implícito el rol y la participación de los científicos. Así, la legitimidad y posicionamiento de una disciplina determinada será enfrentada por los científicos que producen en su interior. La ciencia legitima e institucionaliza y, a su vez, genera impacto en sus ámbitos de discusión y producción. El poder económico y político ha contribuido a generar redes de trabajo interdisciplinarias que reflejan su estrategia al posicionarse como investigaciones autorizadas, por ende, más importantes, puesto que resultan innovadoras y atractivas a los ojos de quienes lucran y comercializan dichos resultados. ${ }^{15}$

La colaboración puede ser nacional o internacional, así como transdisciplinaria y busca impactar en el número de citas que cada autor recibe, beneficiando su visibilidad y de esta forma, su legitimidad, involucrando a los colegios e instituciones desde donde surge la investigación. Luego entonces, se trabaja con la perspectiva de que estas normas y valores beneficiarán y ascenderán de estatus a las instituciones. "Bajo este supuesto, los establecimientos que definen sus misiones, que impulsan formas colegiadas de decisión académica y que introducen sistemas de información y control para hacer más eficiente la administración, tendrían un mejor funcionamiento." ${ }^{.16}$

La legitimación es un concepto principalmente relacionado con aspectos reglamentarios de la vida social. Es un acuerdo que se presenta a modo de relación entre dos partes; una representa la autoridad, gracias a una serie de valores y cualidades que se le atribuyen. La otra son quienes reconocen dicha calidad. ${ }^{17}$ De acuerdo con Scott ${ }^{18}{ }^{18}$ existen tres fuentes de legitimación que serían: científica, social y política, e intelectual. La científica tiene relación con la capacidad propositiva, el

${ }^{15}$ Pierre Bourdieu, El oficio del científico, ciencia de la ciencia y reflexividad, Barcelona, Anagrama, 2003, p. 20.

${ }^{16}$ Germán Álvarez Mendiola, Modelos académicos de ciencias sociales y legitimación científica en México, México, Anuies, 2004, p. 26.

${ }^{17}$ Weber define la legitimidad como "el prestigio de ser ejemplar". Max Weber, Economía y sociedad, México, FCE, 1987, p. 26.

${ }^{18}$ W. R. Scott, Unpacking Institutional Arguments, W. W. Powell \& P. J. DiMaggio [eds.], Chicago, The University of Chicago Press, 1991, pp. 164, 182 (traducción nuestra). 
rigor y la calidad innovadora de la producción planteada en sus investigaciones. La referente a la fuente social y política se remite a la habilidad para la resolución de problemas y a la contención política. El aspecto intelectual es el más alto, el cual se logra cuando las investigaciones de los científicos trascienden y son visibles ante su comunidad y la del propio campo científico, la medición de este aspecto es medido a través de citas.

Según Germán Álvarez, ${ }^{19}$ encontramos una categorización de patrones de reconocimiento dominante en el medio académico:

-El intercambio de bienes de autoridad; sean estos simbólicos o materiales.

La autoridad tiene su fuente en el conocimiento, pero también en la posesión de un certificado que ampare su conocimiento.

- Los bienes académicos son: el prestigio (títulos, premios, reconocimientos).

- El poder (ejecutar contrataciones, y toma de decisiones) y los recursos (salarios, estímulos, financiamientos).

- El intercambio de dichos bienes se da entre personas internas y externas a la institución, que compartan valores, normas y formas de pensar.

- El consenso interno se basa en el acuerdo grupal de los intercambios y en la actualidad, es común que las barreras entre lo interno y externo se abran.

- La legitimidad se convierte en un campo de lucha y de tensiones, en especial cuando es impuesta y no corresponde con los atributos y características esperados. Es decir, también existen casos donde la validación se da de manera implícita y autoritaria.

- Cuando se genera conflicto en alguna de las esferas de la legitimación, ésta se fractura y se corre el riesgo del desprestigio y el bloqueo del funcionamiento del sistema.

Así, como lo indica Michèle Lamont, nos encontramos ante una vinculación de la legitimidad con el poder, que se ejerce a través de un orden jerárquico.

${ }^{19}$ Álvarez Mendiola, op. cit., p. 26. 
Debemos anclarnos al carácter estructural del mismo para estar en posibilidad de darnos cuenta acerca de los fenómenos jerárquicos sociales [...] que indican la incorporación diferenciada de atributos sociales, tal como el prestigio, responsabilidades, el estatus. El poder será definido como la capacidad para imponer la realización de intereses particulares dados en una coyuntura dada. ${ }^{20}$

El aporte de Michel Foucault sobre los estudios del poder con relación al conocimiento es importante. Según Foucault, éste se comprende como algo distinto de las premisas filosóficas y jurídicas que representaban el gobierno de la Ilustración. Por lo tanto, en vez de poseerse y ejecutarse o centralizarse, es visto como una tecnología invisible esparcida a lo largo de la sociedad, vinculada al conocimiento que vigila y castiga. ${ }^{21}$

Otra definición sobre poder, la encontramos en Weber. ${ }^{22}$ Aunque el origen social y las posesiones privadas materiales, el prestigio o la fuerza no son determinantes sobre su ejercicio, éstas tienen un doble estatus y pueden ser utilizadas como tal.

Para Berger y Luckmann existe un proceso de reconocimiento y de integración en el conocimiento, el cual conduce hacia el orden de institucionalización. De esta forma, señalan: "la legitimación produce nuevos significados que sirven para integrar los ya atribuidos a procesos institucionales dispares". ${ }^{23}$

En este sentido, el reconocimiento de un científico es premiado a través de exclusivas condecoraciones. Entre más valorado sea un científico dentro de su comunidad tendrá más autoridad. Otras ventajas que existen de acuerdo al poder y la legitimidad de los privilegiados son: poseer un trato preferente al del resto de sus colegas, acceder a presupuestos económicos más amplios para desarrollar

${ }^{20}$ Michèle Lamont, "Le povoir des intellectuels", en Politique, vol. 1, núm. 1, París, 1982, pp. $19-$ 46.

${ }^{21}$ Michel Foucault, Tecnologías del Yo y otros textos afines, Barcelona, Paidós, 1991, p. 152.

${ }_{22}^{2}$ Weber define el poder como "la posibilidad de que un actor dentro de sus relaciones sociales tenga la posición de realizar su propia voluntad a pesar de las resistencias que surjan”. Weber, op. cit., p. 53

${ }^{23}$ Berger y Luckmann, La construcción de la realidad social, Buenos Aires, Amorrortu, 1995, p. 120. 
sus proyectos e investigaciones, tener puestos directivos o de jefes según sus áreas, viajar e intercambiar continuamente con redes más amplias en un ámbito internacional, representar de forma directa a sus institutos y universidades. En síntesis: ser parte del grupo principal de sus respectivos campos científicos.

La reflexión de Bourdieu respecto a la Teoría de la Praxis afirma: "la legitimidad de la ciencia y el uso legítimo de ésta son, en cada momento, objetivos por los que se lucha en el mundo social y en el propio seno del mundo de la ciencia. Se deduce de ahí, que eso que llamamos epistemología está constantemente amenazada de no ser más que una forma de discurso justificativo de la ciencia." ${ }^{\text {24 }}$

A pesar de las fuentes de legitimación científica descritas antes, las políticas internacionales cambiantes nos exigen también competitividad, responsabilidad, publicaciones, certificación y evaluación de nuestras investigaciones. Álvarez Mendiola simplifica esta perspectiva: "Es decir las fuentes de legitimidad política y social están cambiando hacia formas abiertas y de mercado". ${ }^{25}$ Ante estas medidas, y tiempos de crisis, resulta vital plantear alternativas de respuesta para poder mantener la autonomía de la ciencia, el movimiento de acceso abierto es, sin duda, una vía que en la actualidad incluso los países desarrollados están tomando en cuenta.

Para pasar al siguiente apartado hay que preguntarse: icómo se mide la legitimidad y visibilidad científica?, ¿quiénes están detrás de las evaluaciones de productividad?, ¿cuáles son los dispositivos que se consolidan como los canales óptimos y legítimos de comunicación científica?, ¿cuáles son las disciplinas científicas que producen más?, ¿qué rol están desempeñando las ciencias sociales dentro de la producción global científica?, ¿cómo se evalúa la producción científica?, ¿cuáles son las paradojas de la evaluación científica?, ¿cuál es la contribución de América Latina en la ciencia global? En fin, varias preguntas y debates surgen en torno a la legitimación. Pero véanse previamente los postulados de la Cienciometría como disciplina encargada de la medición científica.

${ }^{24}$ Bourdieu, El oficio del..., p. 20.

${ }_{25}$ Álvarez Mendiola, op. cit., p. 28. 


\section{La Cienciometría a TRAvÉS DE laS baSes DE DATOS}

Estos estudios están situados en un contexto específico donde Michel Callon y Bruno Latour son los precursores de la perspectiva francesa y contribuyeron a la creación del Centre de Sociologie de l'Innovation. Mientras que David Bloor encabeza las investigaciones de la ciencia y técnica en el mundo anglosajón, el trabajo de Bloor se centra en la discusión sobre el apriorismo filosófico en Kuhn y Popper. A través de su obra Conocimiento e imaginario social, publicada en 1971, se perfiló el texto como un estudio del programa fuerte de la sociología del conocimiento. ${ }^{26}$

El objetivo de la Cienciometría radica en identificar leyes y patrones de la actividad científica y tecnológica; para lograrlo se fundamenta en la misma metodología de la ciencia, en la rigurosidad del método científico apoyado en una base cuantitativa. Por ello la Cienciometría se ha planteado generar una cartografía de la ciencia para conocer sus regularidades e interrogarse a sí misma sobre sus producciones. A través de ficheros electrónicos se alcanza una mayor eficacia; éstos albergan la información más importante de los autores, es decir, el título del artículo, el nombre del autor o autores, el instituto de afiliación, el país de producción, las referencias o citaciones.

Los precursores de la Cienciometría americana son el creador de IsI Thompson Eugene Garffield Dereck y Solla Price, a través de los libros Science since Babylon y Little science big science. ${ }^{27}$

\footnotetext{
${ }^{26}$ Su argumento principal señala que las teorías que se consideran como verdaderas o falsas son metodológicamente relativas al objeto estudiado. En esta lógica, la teoría científica es producto de una construcción social; de metáforas colectivas, más que de evidencia, lógica y racionalidad. Peter Slezak tiene un artículo que critica la obra de Bloor, pues aprovecha la reedición de Conocimiento e imaginario social, para rebatir ciertas ideas que le parecen vacías dentro de la obra. Peter Slezak, "Second Look at David Bloor's: Knowledge and Social Imagery", en Philosophy of the Social Sciences, vol. 24, septiembre, 1994, pp. 336-361 (la traducción es nuestra).

${ }^{27}$ Los resultados de Solla Price derivaron en cuatro leyes principales: a largo plazo, la producción científica total se duplica. La curva logística " $S$ " señala que los periodos productivos de la ciencia tendrán, necesariamente, periodos de pasividad y crecimiento limitado. Existe una comunidad científica de élite que detenta la mayor cantidad de artículos publicados, mientras que por el contrario, una masa de investigadores son productivos. La colaboración de científicos que
} 
Los artículos publicados en revistas especializadas y patentes de innovación tecnológica constituyen las fuentes primarias para identificar la producción científica. Esto se afirma ya que los artículos requieren de un exhaustivo proceso que implica la producción de datos innovadores, de la construcción de un orden argumentativo y bibliográfico, y, finalmente, de evaluación por pares ciegos para lograr su publicación. Además, su búsqueda resulta más sencilla al aparecer en publicaciones periódicas que encontramos a través de las revistas. Existen dos métodos de agrupación de los análisis bibliométricos. Estos son: a) de volumen, es decir, aquellos que calculan el impacto de las investigaciones yb) de relación, que deducen aspectos sobre vínculos o interacción entre investigadores y sus áreas consolidadas.

Por otra parte, se tienen varios indicadores de actividad científica. Los más sencillos son los que se contabilizan por el número de publicaciones por área y su productividad. El gráfico de esta información es conocido como curva de Rohtman. Señala el número de investigadores "Y" que han publicado "X" artículos. Esta curva permite confirmar la Ley de Lotka, según la cual sólo unos pocos científicos tienen concentrada la mayor producción de investigación. ${ }^{28}$

El indicador también puede determinar en qué países se concentra la mayor productividad científica y tecnológica en una escala global. En este punto cabe destacar que desde la década de los ochenta son: Estados Unidos, la Comunidad Económica Europea, Inglaterra y Japón los países que encabezan por varios puntos de ventaja la producción científica y tecnológica respecto a regiones como América Latina.

El otro indicador sustancial es el referente a las citas. Cuando se cita un artículo significa que ha sido leído y, por ende, se ha hecho visible para que otros lo citen. También se ha interpretado que su contenido impacta en la producción de conocimientos. ${ }^{29}$

trabajan un mismo tema se agrupan en colegios invisibles. Michel Callon, Jean-Pierre Courtial, Hervé Penan et al., Cienciometría. La medición de la actividad científica: de la bibliometría a la vigilancia tecnológica, Gijón, TREA, 1995, p. 9.

${ }^{28}$ La Ley de Lotka tiene la siguiente fórmula: $\mathrm{Y}=\mathrm{K} / \mathrm{X}$. Michell Callon da el ejemplo que si hay investigadores que han publicado un solo artículo, entonces no habrá más de cuatro investigadores que han publicado cinco artículos y uno solo que ha publicado 10. Loc. cit.

${ }^{29}$ Sin embargo, Callon precisa ciertos inconvenientes que genera la metodología del análisis de citas; en especial para el caso de las bases de datos de Thompson. Entre las más comunes re- 
Por su parte, el grupo de los indicadores relacionales se dividen en primera generación y segunda generación. A través del método de citas conjuntas podemos identificar de forma global quiénes son los autores que citan los investigadores, quiénes colaboran entre sí y, de este modo, podemos conocer los llamados colegios invisibles. En este grupo de indicadores se analizan las firmas conjuntas en un artículo. Al seguir con los indicadores de segunda generación, éstos sí nos pueden proporcionar información sobre los contenidos temáticos de los artículos. El método principal es el de palabras asociadas y se limita a la literatura científica.

Ahora bien, dentro de los aportes clave de Bruno Latour y Michelle Callon está el concepto de redes sociotécnicas. La red deriva del proceso de interacción entre individuos. Los nodos se describen como los puntos que conectan recursos, saberes o informaciones. Una red es más sólida de acuerdo a la cantidad de las alianzas y miembros que la integren. ${ }^{30}$

El análisis de redes se concibe como un sistema que identifica la intercomunicación, el cual se vuelve un medio de integración social. A diferencia de las organizaciones que se articulan jerárquicamente y de forma descentralizada, las redes de colaboración se centran en un nivel de complejidad ligado a la heterogeneidad y diferenciación de los actores participantes. ${ }^{31}$

La Cienciometría ha encontrado una aplicación directa a la evaluación y eficacia de una investigación a través de tres categorías que son: los actores de la

saltan: la forma en la que un mismo autor firma sus artículos, pues en ocasiones anotan sus iniciales, o en el caso de algunos países donde utilizamos dos apellidos, a veces, firmamos con uno solo y otras con los dos. Al capturar la información las bases de datos no diferencian y codifican que se trata de personas distintas. Además las citas que recibe un documento comienzan a aparecer entre seis y dieciocho meses después, por lo que las citas implican ciclos de vida. Otra práctica común es la de la autocitación, la cual provoca que se incremente el número de citaciones, generando un sesgo al análisis. Ibid., p. 9.

${ }^{30}$ El término custers ha sido aportado por Garffield, se trata de un grupo de documentos altamente citados (traducidos como fuentes de investigación) que permiten rastrear las especialidades sobre las que un artículo puede estar relacionado. Indicadores similares pueden obtenerse para el caso de las patentes y el vínculo entre ciencias y técnicas. Ibid., pp. 55-69.

${ }^{31}$ Matilde Luna y José Luis Velasco, "Redes de conocimiento: principios de coordinación y mecanismos de integración", en Mario Albornoz y Claudio Alfaraz, Redes de conocimiento: construcción, dinámica y gestión, RICYT, CYTED, UNESCO, 2006, pp. 15-38. 
investigación, los operadores de la investigación y los sistemas de investigación. Otra dimensión importante es la vigilancia tecnológica, relacionada con la asignación de presupuestos de inversión en materia de ciencia y tecnología.

\section{Thompson Reuters y Scopus, la corriente de la ciencia principal}

Thompson Reuters surgió de la fusión de Thompson Corporation y Reuters Group PLC; es una empresa con fines de lucro enfocada a la venta de información sobre los resultados de la investigación científica, análisis de citación, evaluación de resultados de proyectos de investigación que se generan a partir de un grupo de revistas exigentemente seleccionadas.

Antes llamado IsI Thompson, los orígenes del Institute for Scientific Information se remontan a Eugene Garfield, quien en 1955 publicara un artículo en la revista Science sobre el impacto en la citación. No obstante, el criterio "factor de impacto" "32 fue utilizado por primera vez para cuantificar las publicaciones en la edición de 1963 del Science Citation Index (SCI). ${ }^{33}$

Hoy en día, el Thompson Reuters alberga tres bases de datos de citación dentro de las publicaciones Web of Science; donde existe mayor concentración es en la base de ciencias naturales (Scie) con un total de 8198 revistas; le sigue la base de ciencias sociales (SsCI) con 2813 revistas y, por último, se encuentra el índice de citación de artes y humanidades (AHSI) con 2523 revistas. El total de las publicaciones referidas o citadas en estas bases es de 13534 elegidas de más de 70000 revistas que hay en el mundo.

${ }^{32}$ El factor de impacto se calcula dividiendo el número total de citas que reciben en un año los artículos publicados en una revista en los dos años anteriores, entre el número de artículos publicados en esa revista en esos dos años. (ISI-JCR).

${ }^{33}$ Gualberto Buela-Casal, "Evaluación de la calidad de los artículos y de las revistas científicas: propuesta de factor de impacto ponderado y un índice de calidad", en Psicothema, vol. 15, núm. 1, 2003, pp. 25-35. 
Gráfica 1. Distribución por áreas de acuerdo con la base Web of Science

\begin{tabular}{|lc|}
\hline \multicolumn{2}{|c|}{ Wels of Science } \\
\hline Base & Total de Publicaciones \\
\hline Arts \& Humanities Citation Index & 1523 \\
\hline Science Citation Index Expanded & 8198 \\
\hline Social Science Citation Index & 2813 \\
\hline
\end{tabular}

FuENTE: elaboración propia a partir de Thompson Reuters, 2009.

Cabe destacar que dentro del sci se edita el Journal Citation Report (JCR), publicación anual a partir de la cual es posible calcular el factor de impacto. El JcR se divide en dos secciones: una denominada Sciences y otra Social Sciences. Dentro de la comunidad científica, el factor de impacto ejerce una influencia fundamental y se ha convertido en el índice que por excelencia determina visibilidad y reconocimiento.

En cada una de estas secciones se clasifican las revistas en dos categorías, una por orden alfabético y otra por materias, dentro de ésta se clasifica de mayor a menor factor de impacto. ${ }^{34}$

Gráfica 2. Publicaciones de acuerdo con Thompson Reuters por región

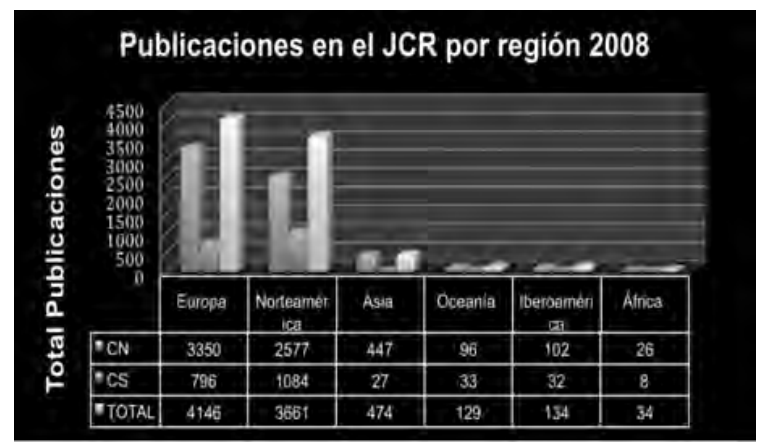

FuENTE: elaboración propia a partir de datos de Thompson Reuters.

${ }^{34}$ Ibid., pp. 25-35. 
En la Gráfica 2 observamos que tanto para el caso de las publicaciones de ciencias naturales como de ciencias sociales entre América Latina y la Península ibérica son sólo relevantes después de la concentración existente en Europa, Norteamérica y Asia.

El dato más importante que nos ha aportado esta perspectiva es el ya referido "factor de impacto". Este índice ha sido útil para conocer la calidad de las publicaciones, sean estos artículos o revistas. ${ }^{35}$

La otra gran base de datos científica puesta en marcha desde 2002 se llama Scopus, es la herramienta más utilizada en Europa. Alberga resúmenes y artículos de cerca de 18000 publicaciones arbitradas, y a diferencia de Thompson admite 350 series de libros, ponencias y conferencias. La mitad de sus documentos provienen de Europa, el resto de Latinoamérica y la región del Pacífico-Sur asiático. Cuenta con expedientes que tienen referencias desde 1996 y hasta 1823. Además también vincula a 435 millones de sitios web científicos. ${ }^{36}$

Otro índice relevante que aclara Scopus lo constituye la citación interdisciplinaria y multidisciplinaria. A través de la citación de artículos que Scopus incluye, se ha consolidado como una fuente importante a la que recurren universidades, institutos internacionales y organizaciones, agencias de información, grupos y empresas transnacionales para tener el listado de las instituciones y científicos más productivos, y a partir de ellos evaluar su investigación y asignar presupuestos por país. Scopus funciona como repositorio privado de la literatura científica de ciencias naturales, tecnología, medicina, y más de 2400 publicaciones para las ciencias sociales, artes y humanidades.

${ }^{35}$ Actualmente, el factor de impacto ha evolucionado como Factor de Impacto Normalizado; un índice que permite evitar sesgos de la relación entre citación y calidad de los científicos provenientes de diferentes áreas. Debido a que sus prácticas de citación y publicación no son iguales, se ha propuesto un índice funcional para todas las disciplinas. "Definimos al factor de impacto normalizado (FIN) como un índice conveniente para evaluar la fuerza de comunidades científicas y la influencia de la investigación individual. El fin es distinguido porque: a) tiene un significado claro y universal que es aplicable con eficacia igual a los individuos que pertenecen a comunidades científicas muy distintas, y: b) éste es sólido contra los casos auto-citación". George Matsas, "What are scientific leaders? The introduction of a normalized impact factor", en Repositorio arxis.org. Brasil, 2008, p. 1 (la traducción es nuestra).

${ }^{36}$ En: www.scopus.com. 
Como respuesta a estos dispositivos, se han venido consolidando diversos proyectos que apuestan por una alternativa a la perspectiva privatizadora, por ejemplo scielo de Brasil y Redalyc de la Universidad Autónoma del Estado de México en América Latina.

\section{Redalyc y SciELO, en acceso abierto para América Latina}

El acceso abierto surge a partir de las declaraciones de Berlín, Budapest y Bethesda. Los argumentos de estas declaratorias se enfocan al acceso abierto; radican en que, por una parte, el conocimiento debe ser diseminado y a su vez autosustentado, permitiéndole abrir nuevas formas de transmisión y democratización del saber acumulado en un solo formato compartible como es Internet.

La Red de Revistas de América Latina y el Caribe (Redalyc) se ha consolidado desde su creación como una iniciativa de acceso libre y gratuita a todo el mundo, y en especial a los científicos de las ciencias sociales de la región latinoamericana. El eslogan de Redalyc: "La ciencia que no se ve no existe" (www.redalyc.uaemex.mx/) reafirma la idea de visibilidad de la producción científica. Redalyc es un portal que auspicia la Universidad Autónoma del Estado de México, donde podemos encontrar artículos completos publicados en ciertas revistas especializadas y debidamente evaluadas, así como datos bibliométricos y ligas a otras páginas de interés de la comunicación científica.

Redalyc alberga un total de 550 revistas científicas y 118917 artículos completos, de los cuales 401 son revistas de ciencias sociales y humanidades, con un total de 82999 artículos. Mientras que para las ciencias naturales y exactas cuenta con 149 revistas y 35918 artículos. ${ }^{37}$ Así es como Redalyc se perfila como una hemeroteca que otorga mayores espacios para revistas de ciencias sociales de la región, lo cual puede ser comprendido como una respuesta ante la tendencia hegemónica de la gran corriente que se inclina hacia los campos de las ciencias duras. Surge como dispositivo alterno que legitima y hace visibles los resultados

\footnotetext{
${ }^{37}$ En www.redalyc.org.
} 
de las investigaciones científicas, de las revistas latinoamericanas, de forma libre y gratuita, al abrir nuevos canales de comunicación académica e institucional.

Finalmente es necesario un análisis del repositorio brasileño scieıo, Scientific Electronic Library Online, por sus siglas en inglés. Como su nombre lo indica es una biblioteca electrónica de las publicaciones arbitradas. Cuenta con una lista alfabética de títulos de revistas, así como por materias y autores de publicaciones provenientes de Iberoamérica. scielo se inscribe en el marco de la política del acceso abierto y permite consultar colecciones de revistas y artículos de texto completo, todas de excelencia académica.

El proyecto lo inició la Fundación de Apoyo para la Investigación del Estado de São Paulo (FAPESP). En colaboración con el Centro Latinoamericano y del Caribe de Información de las Ciencias de la Salud y, recientemente, apoyado por el Consejo Nacional para el Desarrollo de la Ciencia y Tecnología de Brasil. Éste rescata la ciencia producida en los países de la región latinoamericana y funge de canal para hacer visible la ciencia que la corriente principal no toma en cuenta. También funciona como herramienta de evaluación científica, ya que genera indicadores estadísticos de uso y el factor de impacto de las revistas que admite. scielo se define como un repositorio de 623 revistas 239628 artículos y 4794270 citaciones.

Con su creación, scielo contribuyó a que más revistas de las áreas de la salud y la medicina tuvieran sus publicaciones en formato electrónico, abrió las posibilidades de que más lectores en todo el mundo pudieran acceder a sus contenidos. Por otra parte, el portal scielo permitió que las publicaciones de Latinoamérica y en especial de Brasil pudieran incorporarse a una red de revistas científicas de alta calidad y producidas en nuestra región, alternas y descentralizadas de los países desarrollados.

Durante este apartado hemos analizado cuatro bases de datos científicas, cada una con sus particularidades y al mismo tiempo con similitudes, es decir, con espacios electrónicos que permiten almacenar información sobre la producción científica, convirtiéndose en dispositivos mediante los que se legitiman y evalúan a los autores y sus resultados de investigación, ello abre un gran debate sobre el criterio predominante para la evaluación de los investigadores, es decir, el binomio publicación-citación. Éste radica en el impacto de sus trabajos, planteado como la huella 
y repercusión que deja la investigación. Sin embargo, considerar un sólo índice referencial para la evaluación científica resulta limitado y al mismo tiempo excluyente para la mayoría de los científicos latinoamericanos, donde sólo los más prestigiados institutos, universidades, investigadores y alumnos de posgrado cuentan con suscripciones a revistas internacionales indexadas; recientemente se han incorporado a la lectura de revistas electrónicas en acceso abierto.

Cabe destacar que en la región latinoamericana algunos pequeños grupos han llegado a publicar en revistas indexadas por Thompson o Scopus, pero la gran mayoría de nuestros científicos e investigadores no figuran entre estos listados. En este sentido, consolidar espacios incluyentes que legitimen la producción científica regional es todavía una tarea pendiente como científicos en general. Instancias de esta naturaleza permitirían contar con índices más globales y al mismo tiempo incorporarían en esta dinámica a más investigadores. Si se toma en cuenta que, en nuestra región, los recursos con los que se financia buena parte de las investigaciones provienen de fondos públicos, habría que pensar en fortalecer la producción científica desde la iniciativa del acceso abierto.

\section{DisCusión}

A lo largo de su historia, la Ciencia social se ha caracterizado por dar a conocer sus investigaciones a través de libros, esto actualmente se considera como hechos aislados, difíciles de evaluar y de saber si alguien ha leído o citado algún texto propio. Mientras que en la lógica de las revistas indexadas nuestro trabajo es legitimado por pares y por un comité especializado en cada disciplina. Por su parte, las ciencias naturales y exactas provienen de una tradición que ha recurrido a la publicación de los resultados de investigación a través de las revistas. Esta perspectiva nos permite facilitar la comunicación científica de forma global.

En el contexto de la evaluación y el impacto de los trabajos como cientistas sociales en general, los textos aparecen rezagados respecto de los índices que la Cienciometría ha generado, lo que afecta al sociólogo directamente en el reconocimiento y la legitimidad. Ahora bien, en lo que concierne a la región latinoamericana, su participación en la denominada corriente de la ciencia principal se 
encuentra bastante limitada, situación que ha derivado en el surgimiento de nuevos indexadores de la producción científica regional, quienes sabiendo aprovechar las nuevas Tecnologías de Información y Comunicación han juntado e incorporado las publicaciones con mayor calidad en la región, convirtiéndose así en medios legitimadores y comunicadores para la evaluación científica. Por lo que cabe preguntar: ¿como cientistas sociales latinoamericanos conocemos estos indexadores? ¿Cómo podemos incrementar nuestra participación en estas publicaciones? ¿Qué podemos hacer desde las ciencias sociales para revertir las leyes del mercado en la lógica de la producción científica? Las respuestas no se tienen aún, pero desde este artículo, buscamos iniciar los debates y abrir la discusión en torno a esta temática.

\section{CONCLUSIONES}

En este artículo hemos realizado un vasto recorrido para explicar cómo surge la legitimidad de la ciencia. Analizamos la denominada "corriente de la ciencia principal", la cual es conducida por la perspectiva de los países desarrollados.

Esta perspectiva ha provocado que surjan nuevos espacios y dispositivos de legitimación; para el caso de América Latina distinguimos como ejemplos a Redalyc y scieto, proyectos de acceso abierto. El primero gestado desde la Ciencia social ha promovido mayores espacios para las publicaciones especializadas en estas áreas. Mientras que scielo surgió de manera focalizada en Brasil, desde la medicina, y ha incorporado gradualmente a otras disciplinas y países. Estas dos bases de datos han utilizado las Tic's para hacer de la ciencia una esfera más abierta y democrática y, al mismo tiempo, han devenido en legitimadores y evaluadores de la producción regional de América Latina.

La evaluación científica hasta nuestros días se fundamenta en índices numéricos, los cuales sin duda son importantes, pero con la incorporación de valores de tipo más cualitativo, podríamos tener un panorama más global sobre el estatus de la investigación de los científicos. De otra forma, los investigadores de nuestra región se preocupan por la acumulación de cantidad de puntos y números que los institutos de ciencia y tecnología de cada país otorgan, descuidando la calidad de sus investigaciones y el valor de la labor científica. 
La jerarquización que otorga el Citation Index beneficia al campo de las ciencias naturales y en especial a los laboratorios del mundo anglosajón. Es en este punto, cuando la función de nosotros como sociólogos que estudiamos la ciencia, desde América Latina, queda en tensión. Podemos comprobar entonces que la ciencia no es una esfera que se encuentra al margen de la sociedad, más aún, no es un dominio perfecto que se legitima de manera independiente. La propia evaluación por pares es un proceso social, que hasta hoy se ha constituido como garante de la calidad de nuestros trabajos y que, sin embargo, puede ser complementado con nuevos índices y mediciones que los propios científicos pueden proponer y construir. Más allá de la crítica marginal que siempre hemos hecho, de manera desafortunada, la Ciencia social no se ha percatado de la responsabilidad y posibilidad que tiene respecto de su propio posicionamiento y legitimidad, por lo que, además de los índices cualitativos que la Cienciometría ha desarrollado, se podrían proponer vías para una evaluación más amplia que permita eliminar los procesos paradójicos de los mecanismos sociales, en especial en la Ciencia social. Tal vez comenzar con un consenso sobre cuáles indicadores son los apropiados para nuestras disciplinas nos permita mejorar la evaluación, credibilidad y legitimidad como científicos.

Recibido: 24 de marzo, 2010. Aceptado: 27 de mayo, 2010.

\section{BibLIOGRAFÍA}

ABello, RAImundo, Páez JaVIER et al., "SSon la ciencia y la tecnología un instrumento de desarrollo? Un análisis para el caso de América Latina”, en Investigación y Desarrollo, vol. 9, núm. 1, Barranquilla Universidad del Norte, julio, pp. 372-386. 
AleXander Jefreme, Real Civil Societies. Dilemmas of Institutionalization, SAGE, 1998, 256 pp.

Álvarez Mendiola, Germán, Modelos académicos de ciencias sociales y legitimación científica en México, México, ANuies, 2004, 443 pp.

BERGER, P. \& LuckMANn T., La construcción social de la realidad, trad. de Silvia Zuleta, Buenos Aires, Amorrortu, 2001, 233 pp.

Bloor, Peter, Conocimiento e imaginario social, trad. de Emmanuel Lizcano y Rubén Blanco, Barcelona, Gedisa, 1998, 286 pp.

Bourdieu, Pierre, Les usages sociaux de la science, París, INRA Éditions, 1997, 82 pp. , Algunas propiedades de los campos. En sociología y cultura, trad. de Martha Pou, México, Grijalbo/Conaculta, 1990, 228 pp. , Homo Academicus, trad. de Peter Collier, Londres, Cambridge Polity Press, 1988, 433 pp. , El oficio del científico. Ciencia de la ciencia y reflexividad, Barcelona, Anagrama, 2003, 212 pp.

Buela-Casal, Gualberto, "Evaluación de la calidad de los artículos y de la revistas científicas: propuesta de factor de impacto ponderado y un índice de calidad", en Psicothema, vol. 15, núm. 1, 2003, pp. 25-35.

Callon M., J.P. Courtial, H. Penan et al. Cienciometría. La medición de la actividad científica: de la bibliometría a la vigilancia tecnológica, Gijón, TREA, 1995, 110 pp.

Foucault, Michel, Tecnologías del Yo y otros textos afines, Barcelona, Paidós, 1999, 150 pp.

GiBBS, W. WAYT, "Ciencia del Tercer Mundo", en Investigación y Ciencia, núm. 231, 1995, pp. 70-79.

Hurd, Julie M., "Scientific Communication: New Roles and New Players", en Emerging Issues in the Electronic Environment: Challenges for Librarians and Researchers in the Sciences, Londres, The Haworth Information Press, 2004, 257 pp.

Lamont, Michèle, "Le povoir des intellectuels", en Politique, vol. 1, núm. 1, París, 1982, pp. 19-46. 
Latour Bruno, Science in Action: How to Follow Scientists and Engineers through Society, Open University Press, 1987, 274 pp.

Luna, Matilde y José Luis Velasco, "Redes de conocimiento: principios de coordinación y mecanismos de integración", en Mario Albornoz y Claudio Alfaraz, Redes de conocimiento: construcción, dinámica y gestión, París, RICYT, CYTED, UNESCO, 2006, pp. 15-38.

MatSAS George, "What are Scientific Leaders? The Introduction of a Normalized Impact Factor". En Repositorio arxis.org, Brasil, 2008, pp. 1-4.

Martin, Olivier, Sociologie des sciences, París, Armand Collin, 2003 (Col. de Sociología, 128), 128 pp.

PollaK, Michael, "Projet scientifique, carrière professionnelle et stratégie politique", en Actes de la recherche en Sciences Sociales, vol. 55, núm. 55, 1994, París, pp. 54-63.

Scoтt, W. R., "Unpacking Institutional Arguments", en W. W. Powell \& P. J. DiMaggio [eds.], The New Institutionalism in Organizational Analysis, Chicago, The University of Chicago Press, 1991, pp. 164-182.

WaLSH John \& SADAO NAGAOKA, "Who Invented?: Evidence from the Japan-US Inventor Survey", en RIETI Discussion Paper Series, vol. 9-E, núm. 34, pp. 1-70.

Weber, Max, Economía y sociedad, vol. 1, México, FCE, 1993, pp. 12-37.

SITIOS DE INTERNET:

Red de Revistas de América Latina y Caribe. En www.redalyc.uaemex.mx Thompson Reuters. En sitio web: http://www.thompsonreuters.com/

Scopus: http://www.scopus.com. 